\title{
Interactive Design of Free Sharing APP Based on Mobile User Requirements Analysis
}

\author{
Chen Wei*, Bu Qing Kai* \\ *School of Electronic and Information Engineering, QingDao University \\ QingDao,Chinar609339717@qq.com
}

Keywords: User Demand; Free Sharing; Second-hand Goods; Mobile Internet

\begin{abstract}
In recent years, people's living standards have been improved. The constant updating of things has kept people in the pursuit of fashion, a phenomenon that is evident in young groups. For the items that have been changed, they are often shelved or discarded, and the value of the goods is not fully represented. Therefore, it is very important to reuse the second-hand goods. Under the premise of the rapid development of modern mobile Internet technology, mobile devices have added more and more convenience and fun to our life. On this basis, we design an iOS mobile phone application based on the user's demand analysis and mobile application experience, which is the main function of commodity presentation and exchange. The purpose of this study is to have the goods idle and second-hand goods needs of the user to provide a platform for free and mobile users, the Internet technology and the demands of daily life with convenient goods maximum value. On the Mac platform, the design and implementation of APP functions are displayed on the iOS mobile phone terminal, and the implementation principle and related features of iOS system software are analyzed, which reflects the theme that science and technology can make human life better.
\end{abstract}

\section{Introduction}

In the era of rapid development of mobile Internet technology, mobile devices can connect to network services anywhere. Based on mobile phone hardware improvement, a collection of mobile phone application of advanced communication technology and network service technology in changing people at the same time on the mobile phone cognition, also in daily life, learning, work and entertainment plays an irreplaceable role[1]. The design of a second-hand product "Give" platform based on mobile Internet meets the current theme of saving resources and sustainable development.

The design concept of Sharing App comes from the story of "A needle for a car", extracting and analyzing the user's demand information from a large number of user experience data. The user experience and needs, taking into account the app interface interaction design and functional design, to build a free exchange and gift platform. To achieve the user's registration, login, commodity publishing and browsing, message and chat functions. The combination of mobile Internet technology and the needs of users in daily life reflects the theme of "science and technology make human life better".

\section{The Research of User Demand Analysis}

\subsection{Demand Status}

With the development of The Times and the quickening pace of people's daily life, the frequency of daily necessities has also become faster. For students, graduates leave the campus when there will be a lot of things you can not go, throw a pity. In fact, these things can give mentees, helping to make up. For residents of the District, there is no need to have items at home, then hope that residents of the district need to come directly to take, not only to idle goods can continue to play value, but also to know more friends[2]. Whenever this time, we want to have such a platform, you can easily handle idle items. In fact, for 
someone you don't need, someone who needs it might be near you.

\subsection{Investigation and Analysis of Idle Goods}

An online questionnaire survey was conducted on a second-hand commodity trading platform in China as shown in the figure.

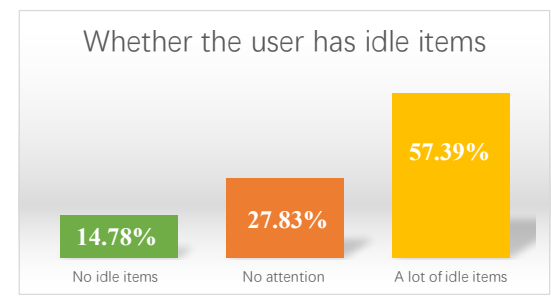

Fig. 1. Data statistics of idle goods

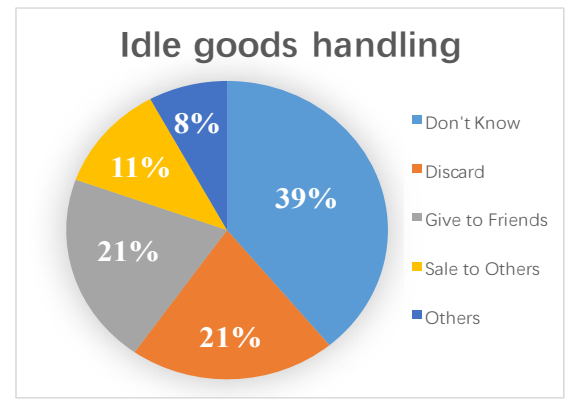

Fig. 2. Idle goods handling

From the analysis of data in Figure 1, we can see that $57.39 \%$ of the residents have idle items at home. In Figure 2, we can see that more than $85 \%$ of the residents who have idle items can not find effective ways to deal with the idle goods.

\subsection{APP Concept}

"Give" is a centralized free goods mobile platform app, introduced the "free" core concept, for the user's idle goods to play a greater value.

The development of the real economy today, the material is extremely rich, resulting in the increase of idle goods, and the value of these idle goods did not reflect. But these unused items are valuable[3]. Just not in the hands of those in need, and no one is willing to share this value with each other. So, APP named "Give" offers a platform for free sharing, giving away unwanted items to people in need, and rediscovering the value of unused items.

\section{User Interaction Experience Analysis}

\subsection{User Experience}

The rapid development of mobile terminal APP is an indisputable fact that the development and access of various clients have become the norm. We can see from the general trend that the demand of users is increasing. User churn is inevitable if developers fail to provide users with better experience with APP. From smartphones to tablets, we can clearly see the rapid growth in functionality, design, and potential of the APP involved[4]. In addition to the requirements of the user experience, but also need to provide sufficient and sufficient APP influence, enhance attention. So that users gradually transformed into the true sense of "consumers".

\subsection{Interface Design Pattern}

The fuzzy background is in line with the current design of flattening and modern wind. It is beautiful enough to match the popular elements such as ghost buttons to enhance the user experience. From a design point of view, it is not only easy to implement, helps to design, avoid complex design, and can also reduce design costs[5].

In the app in the design of navigation, clear typography, clean interfaces, and pleasing APP design are what users like and look forward to most at the moment. A simple down menu and sidebar will be more in tune with trends than fancy and fancy menu designs.

Simple beauty is the most popular design idea in recent years, and a simpler color scheme fits the train of thought. And today's users prefer a subtle and textured interface. Neat and clean interface color schemes are overriding those gorgeous and flamboyant color schemes[6].

\section{App Module Design}

The app named "Give" design pattern is similar to the O2O model, which belongs to mobile e-commerce. The main functions include giving and demanding items between users, personal registration, login, content publish and message reply. 
APP has five main interfaces, topics for GIVE, WANT, PUBLISH, MESSAGE and CENTER. The interface system mainly uses two Storyboard to construct. Login.storyboard including login registration module, is responsible for the realization of user registration and login interface design. Main.Storyboard is responsible for the construction of other interfaces, including the five main interfaces of the UINavigation as the controller. GIVE and WANT two interface design patterns based on user experience, so that users can easily browse second-hand goods information. RELEASE interface, publish unused items and fill in relevant information. The MESSAGE interface display comments private message. The CENTER interface contains personal nicknames, accounts, avatars and other information. The design of the system function module is shown in Figure 3.

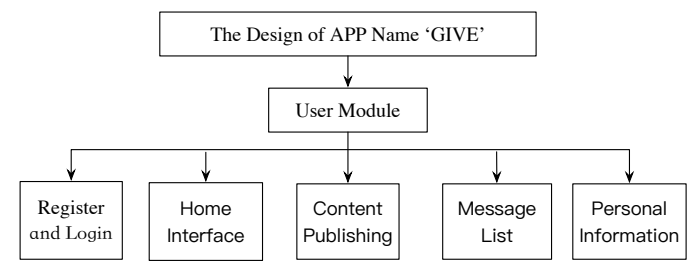

Fig. 3. System Function Module Design

\subsection{Register and Login Module}

The user registers and sets the password by phone number verification. Only when a user login to the relevant operation, when not login will remind the user login.

When a user enters a personal center, he first enters a login screen. The center of the interface is the user name and password input box, click the registration button, you can jump to the registration interface. When the user name and password are correct, click the login button to enter the personal center page. The user name and password are not entered or the error will raise an error prompt. As shown in Figure 4, the user login flow chart.

When the user does not account number, you need to click the registration button in the lower right corner of the login. You need to fill in your cell phone number, password in registration interface, and received verification code. When registration is complete, the first login will have boot operation. Ask the user to set up the nickname, avatar and other information.

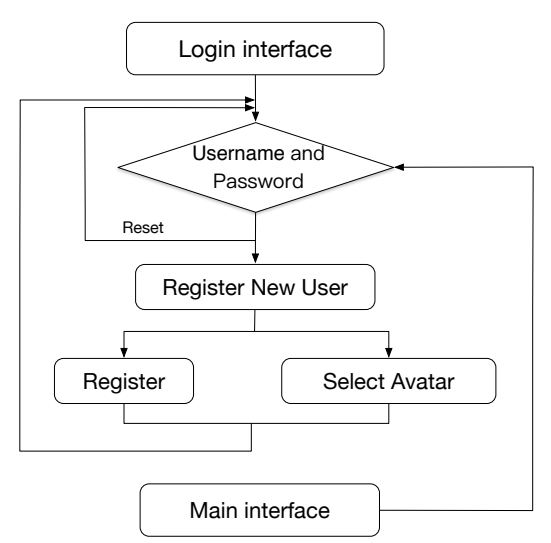

Fig. 4. Register and Login Flow Chart

\subsection{Home Module}

Home page is the core module of app, which mainly displays the information of second-hand articles issued by users. The module mainly has "Give" and "Want" two primary interface, and the corresponding interfaces display the user's information and ask for the goods information. The upper left corner of the interface shows the geographical location, you can click on the label to jump to the two level geographical location interface, select the automatic status or manual position. The upper right corner shows the search button, click on the label to jump to the contents of the search interface, enter the key information in the search box, then determine the search load stored in the database according to the user requirements of the search items of information[7].

"Give" and "Want" two interfaces are made up of UITableView, using the custom designed Cell layout and pulling data loaded using the MJRefresh framework. Custom cell consists of three parts, from top to bottom: user information part, commodity information part, evaluation part.

The top user information section displays the user's Avatar and nickname from left to right. Click on the avatar to jump to the user's personal details interface. The information section of the intermediate commodity is a picture of the product and a detailed description of the information, and the picture is displayed in full screen when clicked. The bottom portion of the evaluation shows the location, message, and number of items posted. Click the "Praise" button, corresponding to "Praise" the increase in the number. Click on each cell to jump to the details of the article 
interface, where you can browse the full information of the goods. Below is other users' comments and comments, messages and evaluation messages will be automatically notified to the release of the user, and users in the main interface, message module will receive other users' message and evaluation information[8].

\subsection{Content Publishing Module}

Users must login before they can publish content. When a user releases an article, it needs to fill out the published information. The user is not only the giver of the goods, but also the purchaser of the goods. Click on the release button at the bottom of the app home page to create a presentation and request interface below the interface. Click the "Give" button, it will pop up an object registration interface, fill in the name of the goods, photos, detailed description, location, contact information and other information, and finally publish the goods. Click the "Want" button, just fill in the user's needs and practices. Successful release information will be displayed on the presentation request interface. Figure 5 shows the flow chart.

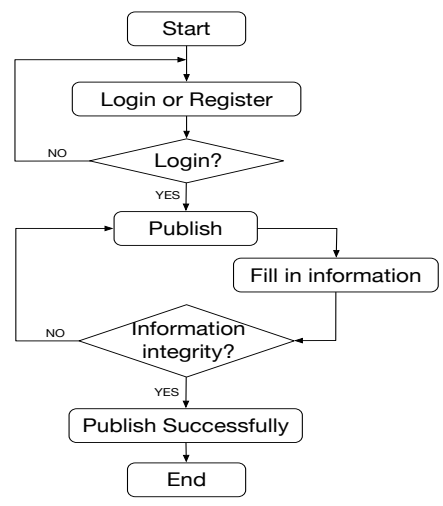

Fig. 5. Content Release Flow Chart

\subsection{Message Lis Module}

The message list module uses UITableView as the primary interface controller. Message, message evaluation, private letter three parts using a custom Cell layout. The message is the message received by other users under the published content. Evaluation information is the evaluation of goods by the recipient after the gift is finished. For other users to publish news is the content transfer way to chat messages.

\subsection{Personal Module}

The personal center module includes three parts: personal information, user labels and grades, and system settings. The personal information section uses the custom UIView to display user nicknames, avatars, uploaded personal pictures, and published secondary commodity information. The other two uses the grouping function of the UITableView. The first group is user tags and grade sections, setting personalized tags that are often used by the user. The second is the system settings, including the cleaning of the software cache, the notification of the system, the introduction of the software and so on.

\section{Summary}

The status quo through background analysis, iOS system based on APP is free to share items, the free sharing of ideas, according to the user demand for everyday life as a foundation of the end of the mobile phone software requirements. In practical applications, it can fully help the people in need. Play the use value of second-hand goods, make people's life more convenient, experience science and technology, make human life more beautiful one theme. The software interface has the advantages of simple operation, clear logic, perfect function structure and systematic design idea, which can enlighten the design and development of other similar APP software.

\section{REFERENCES}

[1] Qin Caijie, WEI ZhongTian, "Research on Key Technology of O2O Clothing Shopping APP Based on iOS Platform," Journal of Yibin University, Vol. 16, No. 12, pp: 56-59.

[2] Chen Jie, "The Design of Shopping App Based on 1OS System," Journal of Office Automation, Vol. 330, pp: 42-44.

[3] Tang Qitao,Zhang Yan,Peng Lihong," Research and Design of Tourism APP Based on Ios," Computer Knowlegde and Technology,Vol. 11, No. 25, September 2015, pp: 39-41.

[4] Ren Peihua,Xuan YuRu, “Campus Flea MarketMobile Terminal App Based on Android," Computer \& Digital Engineering,Vol. 44, No. 11, 2016, pp: 2276-2279.

[5] Wu JuLiu,Wu MaoXue,Lv FangYuan. “ Design and Development of PHP Campus Second-hand Commodity Trading Platform,”. Science \& Technology Information,Vol. 12, No. 13, 2014, pp:33-34.

[6] Tu Xiaoyun.“ Design and Implementation of the Trading Platform for Secondhand Goods," . Fujian Computer, 2011, Vol. 2 7, No. 8, pp: 115-116.

[7] Lu JinSheng, Chen Qing,Chen XiJing, “App Interface Study on How to Improve User Experience," The 7th International Conference on Computer Science \& Education (ICCSE 2012) July 14-17, 2012. Melbourne, Australia, pp: 726-729.

[8] Xu Lei, Wang Xuan, "Research on Dual-platform-based Wireless App Developement and System Implementation,” 2013 Inte ational Conference on Mechatronic Sciences, Electric Engineering and Computer (MEC) Dec 20-22, 2013, Shenyang, China, pp: 1912-1916. 